\title{
Establishing free-living flatworm Macrostomum lignano as a model to study links between regeneration and cancer
}

\author{
M. Biryukov ${ }^{1,2 *}$, I. Sukhikh ${ }^{1}$, V. Vavilova ${ }^{1}$, K. Ustyantsev ${ }^{1}$, E. Berezikov ${ }^{1,3}$ \\ ${ }^{1}$ Institute of Cytology and Genetics SB RAS, Novosibirsk, Russia \\ ${ }^{2}$ Novosibirsk State University, Novosibirsk, Russia \\ ${ }^{3}$ European Research Institute for the Biology of Ageing, Groningen, The Netherlands \\ *e-mail:mikebirykov@yandex.ru
}

Key words: Macrostomum lignano, flatworm, carcinogens, toxicity, lethality

\begin{abstract}
Motivation and Aim: The restriction of regeneration is thought to evolve as a measure against cancer. Highly regenerative flatworms do not have cancer, suggesting that they evolved some other regulatory mechanism to keep their cells under control while allowing the process of regeneration. However, it is possible to induce carcinogenesis in flatworms with chemical mutagens [1, 2]. Macrostomum lignano is a free-living flatworm rapidly emerging as a model organism to study regeneration and stem cell biology [3]. $\mathrm{CdSO}_{4}$ and 12-O-tetradecanoylphorbol 13-acetate (PMA) are established chemical carcinogens with known tumorigenic effect in planarians $[1,2]$. The aim of this study is to find optimal concentration for $\mathrm{CdSO}_{4}$ and PMA induced carcinogenesis in $M$. lignano, which should be a first step in dissecting conserved regulatory gene networks interplayed between regeneration and cancer.

Methods and Algorithms: M. lignano worms were grown in artificial seawater enriched with $\mathrm{f} / 2$-medium. Various concentrations of $3 \mathrm{CdSO}_{4} \cdot 8 \mathrm{H} 2 \mathrm{O}, \mathrm{PMA}$ (diluted in DMSO), and their combinations were tested for toxicity. Twenty adult worms were continuously exposed to various concentrations of the carcinogens for 2 weeks (the medium was refreshed weekly), and the percentage of lethality was calculated for each of the conditions.
\end{abstract}

Results: Thirty different carcinogen concentrations were tested for CdSO4, PMA, and

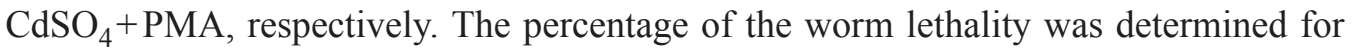
each of the conditions. The toxicity was established as lethal concentration $50\left(\mathrm{LC}_{50}\right)$. Concentrations of the carcinogens suitable for chronic exposure of worms and tumor growth induction were selected.

Conclusion: The results obtained can serve as a starting point to the carcinogenesis study in M. lignano.

Acknowledgements: The project was supported by the Russian Foundation for Basic Research (Grant No. 18-04-01011).

\section{References}

1. Hall F. et al. (1986) Neoplastic transformation in the Planarian: I. Cocarcinogenesis and tiistopathology. Journal Experimental Zoology. 240:211-227.

2. Voura E.B. et al. (2017) Planarians as models of cadmium-induced neoplasia provide measurable benchmarks for mechanistic studies. Ecotoxicology Environmental Safety. 142:544-554.

3. Wudarski J. et al. (2017) Efficient transgenesis and annotated genome sequence of the regenerative flatworm model Macrostomum lignano. Nature Communications. 8:2120. 